\title{
Preserved Implicit Learning on both the Serial Reaction Time Task and Artificial Grammar in Patients with Parkinson's Disease
}

\author{
Jared G. Smith (smithjare@scs.vuw.ac.nz) \\ School of Psychology, Victoria University of Wellington, \\ PO Box 600, Wellington, New Zealand
}

Richard J. Siegert (Richard.Siegert@vuw.ac.nz)

School of Psychology, Victoria University of Wellington, PO Box 600, Wellington, New Zealand

John McDowall (john.medowall@vuw.ac.nz)

School of Psychology, Victoria University of Wellington, PO Box 600, Wellington, New Zealand

David Abernethy (david.abernethy@clear.net.nz)

Wellington School of Medicine, University of Otago, PO Box 600, Wellington, New Zealand

\begin{abstract}
Thirteen non-demented patients with Parkinson's disease (PD) were compared with age matched controls on two standard tests of implicit learning. A verbal version of the Serial Reaction Time (SRT) task was used to assess sequence learning and an artificial grammar (AG) task assessed perceptual learning. It was predicted that PD patients would show implicit learning on the AG task but not the SRT task, as motor sequence learning is thought to be reliant upon the basal ganglia which is damaged in PD. Patients with PD demonstrated implicit learning on both tasks. In light of these unexpected results the research on SRT learning in PD is reconsidered, and some possible explanations for the sometimes conflicting results of PD patient samples on the SRT task are considered. Factors which merit further study in this regard are: The degree to which the SRT task relies on overt motor responses; the effects of frontal lobe dysfunction upon implicit sequence learning; and the degree to which the illness itself has advanced.
\end{abstract}

Current theoretical accounts of human memory draw an important distinction between implicit and explicit learning processes (e.g., Squire, 1994; Squire \& Zola, 1996). Explicit (or declarative) learning and memory is characterized by the acquisition and retrieval of information accompanied by awareness of the learned information and its influence. Implicit learning refers to similar acquisition without awareness of the learned information or its influence. Such learning occurs in situations and tasks whereby the ability to consciously or deliberately recall the episode in which learning took place, or to describe the rules underlying the task, typically fall well behind the level of performance. It is thought that explicit learning is dependent upon medial temporal lobe and diencephalic brain structures, while habit learning and implicit skill learning is closely associated with neostriatal structures such as the basal ganglia (Squire, 1994; Squire \& Zola, 1996).

One striking characteristic of implicit learning has been its demonstrable robustness even in the face of quite major brain damage. For example, using the serial reaction time (SRT) task researchers have shown implicit learning to be preserved in normal ageing (Howard \& Howard, 1989), Korsakoff patients (Nissen \& Bullemer, 1987), closed head injury patients (McDowall \& Martin, 1996) and Alzheimer's disease (Knopman \& Nissen, 1987). However, the fact that the very brain structures thought to be closely associated with certain forms of implicit learning are the most impaired by Parkinson's disease (PD), makes PD of special interest for implicit learning researchers. The characteristic neuropathology of PD includes marked degeneration and atrophy of the basal ganglia and substantia nigra, particularly the caudate nucleus or the neostriatum (Knight, 1992). In the present study we were interested to compare the performance of PD patients with controls on two tests of implicit learning: the serial reaction time (SRT) task and the artificial grammar (AG) task.

In the SRT task participants respond as quickly as possible to the presentation of an asterisk on a computer monitor. The asterisk can appear at any one of several different locations and participants must respond by pressing a key which corresponds to the spatial location of the asterisk. Unknown to the participants, the location of the stimulus follows a sequence which is repeated over a number of trials. Sequence learning is assumed to occur when, over the course of successive trials, the reaction times (RT) of participants decrease significantly and when there is a significant increase in the RT of participants upon the 
administration of a block of trials where the position of the asterisk is random. Of particular interest, is that while participants display significant learning over trials, they are often unaware that such learning has occurred and mostly unable to correctly report the actual sequence followed (Nissen \& Bullemer, 1987).

In the first study of PD patients using the SRT task Ferraro, Balota and Connor (1993) reported that nondemented individuals with PD showed less sequence specific learning than healthy controls. Similarly, PascualLeone et al. (1993) found patients with PD acquired some SRT procedural knowledge although its degree was less than in healthy volunteers. However, perhaps the clearest evidence for an implicit visuomotor learning deficit in patients with basal ganglia dysfunction comes from a study by Jackson et al. (1995). The authors found no significant SRT learning in PD patients and concluded that the results suggest a role for the basal ganglia in SRT learning or the expression of serially-ordered action. Westwater et al. (1998) employed a verbal version of the SRT task, designed to minimize the influence of the motor symptoms of PD, and reported similar results. In summary, there is a growing number of studies suggesting that implicit learning in PD, at least as measured by the SRT task, is reduced or impaired in people with $\mathrm{PD}$.

AG learning involves presenting participants with a set of rule-governed stimuli (typically cards consisting of letter strings belonging to a finite-state grammar) for observation, and asking them to commit the letter strings to memory. The set of stimuli typically consists of exemplars which cover the entire range of transitions of the grammar, providing exposure to all the rules of the grammar albeit in an indirect fashion. On completion of the orientation task, participants are informed of the existence of a complex grammatical system governing the stimuli presented. Participants are then shown a new set of cards, only half of which conform to the grammar, and asked to decide whether each item conforms to the structure of the grammar. The assumption behind this paradigm is that tacit knowledge, which is abstract and representative of a complex grammar system, can be learnt independently of conscious efforts (Reber, 1989).

One important theoretical issue for the study of implicit learning concerns the degree to which different types of implicit learning are separate or dissociable both functionally and at an anatomical level. For instance, while formally similar to habit learning paradigms such as the SRT task, AG participants typically evidence abstract knowledge about a complex rule system on grammaticality tests, while the measure used in SRT tasks is reaction time, which is more likely to tap visuomotor knowledge (Seger, 1998). The examination of abstract judgment-linked learning (e.g., AG learning) and visuomotor learning in a group such as PD patients, where brain structures assumed to be involved in implicit learning processes are damaged, provides a method to investigate the possibility that these forms of implicit learning may be independent.

While several studies have examined the performance of PD patients on the SRT task (and generally found deficits or impairments), to our knowledge only two published studies have reported using the AG task with PD patients. Thierry, Peigneux and Van der Linen (1998) observed the same level of performance in controls and patients with $\mathrm{PD}$ on initial trials which suggested preserved AG learning in PD, and more broadly, that the basal ganglia may not be crucially involved in the rule-extraction mechanisms engaged in AG learning. Recently, Reber and Squire (1999) investigated the ability of patients with PD to learn AG in both a standard condition and a letterset transfer version of the task. They observed learning under both conditions and concluded that the learning of AGs appeared not to depend on the integrity of the neostriatum. They also commented that the dissociation between SRT and AG performance in patients with PD relies upon comparisons across studies, and that a dissociation within the same group of patients would be even stronger evidence.

The finding that patients with $P D$ exhibit intact $A G$ learning but show impairment on SRT tasks suggests implicit learning is not a single entity and that different neural systems may mediate performance on particular implicit learning tasks. In the present study we set out to compare the performance of a group of patients with PD on the SRT task with their performance on an AG task. The verbal version of the SRT task replaced the standard button-pressing response with a vocal response in an attempt to reduce the motor component of the task. We hypothesized that patients with PD would show impaired performance on the SRT task but not on the AG task in comparison to healthy controls.

\section{Method}

\section{Participants}

Participants consisted of 14 patients with PD recruited from the Neurology outpatients' service of Wellington Hospital, and 14 volunteers from the community who served as healthy controls. The diagnosis of PD was confirmed by a senior staff neurologist. One member of the PD group scored below the standard cut-off of 24 points on the MiniMental Status Examination (MMSE; Folstein, Folstein, \& McHugh, 1975), used as a screening measure for abnormal cognitive decline, and was excluded from further analyses.

The PD group comprised eight males and five females, with a mean age of 66.42 years (range $=37$ to 79 years). In the control group, eight were male and six were female, and the mean age was 68.36 years (range $=53$ to 74 years). Each of the patients with PD in the present study fell within the early to middle/late stages of severity as assessed by the Hoehn and Yahr (1967) degree of clinical disability scale. Ten of the patients were in Stage Two (bilateral midline involvement without loss of balance), two were in Stage Three (first signs of impairment in equilibrium, significant slowing of body movements), and one was in Stage Four (fully developed PD, still able to stand and walk, but markedly incapacitated). At the time of testing all patients with PD were under the care of a neurologist and all but two were receiving anti-Parkinsonian medication. None had a history of head injury within the preceding ten years, or had a history of alcohol abuse, stroke or epilepsy, and all 
subjects had normal or corrected to normal vision. The administration of a standardized measure of depression indicated an absence of depression for all participants.

\section{Materials}

All participants were administered the National Adult Reading Test (NART; Nelson \& Willeson, 1991) to compare performance on intellectual ability. Additionally, the Controlled Oral Word Association Test (COWAT; Benton \& Hamsher, 1976) was administered in order to assess verbal fluency. There were no significant group differences on variables of age, gender, or number of years spent in formal education. A summary of the group demographics is displayed in Table 1.

Table 1. Demographic Data.

\begin{tabular}{lll}
\hline Measure & \multicolumn{1}{c}{ PD } & $\begin{array}{c}\text { Controls } \\
\mathrm{M}(\mathrm{SD})\end{array}$ \\
\cline { 2 - 3 } & & \\
\hline Age (yrs) & $66.4(11.0)$ & $68.3(8.4)$ \\
Education (yrs) & $12.2(2.7)$ & $12.3(2.6)$ \\
MMSE & $27.3(2.0)$ & $29.0(1.3)$ \\
COWAT & $33.6(12.2)$ & $47.6(2.1)^{*}$ \\
NART & $116.5(5.0)$ & $122.0(10)^{*}$
\end{tabular}

$* \mathrm{p}<.05$

Note: MMSE = Mini Mental Status Examination; COWAT $=$ Controlled Oral Word Association Test - age corrected scores: NART $=$ National Adult Reading Test, expressed as a Wechler Adult Intelligence Scale - Revised full scale equivalent.

\section{Apparatus and Procedure}

All participants were tested individually beginning with the NART, followed by the MMSE. Following this participants either completed the SRT, the COWAT, and then the AG task, or performed these three tasks in reverse order. The ordering of these three tasks was counterbalanced within both the PD patient group and the control participant group.

SRT Task. The SRT task was a verbal version of the classic SRT task, as devised by Nissen and Bullemer (1987), replicating the SRT task used by Westwater et al. (1998) (refer to Westwater et al. for a more detailed description of the procedure). Briefly, all participants completed five blocks of trials, each consisting of 100 trials. In each trial a stimulus (an asterisk) appeared in one of four positions along the bottom of a computer monitor. In the first four blocks the asterisk appeared in a sequential manner (the 10item sequence used in Nissen and Bullemer (1987)). In the fifth block the location of the asterisk was determined pseudorandomly. All participants were asked to respond as quickly as possible to the location of each stimulus by saying aloud the number corresponding to its location. Upon a response the stimulus disappeared and $400 \mathrm{~ms}$ later the next stimulus appeared in one of the other locations. At the conclusion of the task all participants were asked whether they noticed anything about the nature of the stimuli. Although some participants reported being aware of some form of pattern to the stimuli, none were able to correctly reproduce it when asked to do so.

Artificial grammar task. Grammatical letter strings were generated from a finite-state Markovian rule system identical to that used by Dienes, Broadbent, and Berry (1991). This structure was used to generate both 23 training and 23 test items, each three to six letters in length. Twenty-three non-grammatical test items were also generated from the rule system by substituting an inappropriate letter for an appropriate letter in an otherwise grammatical string. Each letter string was presented on a $7.5 \times 12.7 \mathrm{~cm}$ index card.

The procedure for the training and testing phases closely followed the standard AG procedure and is fully described by Dienes et al. (1991: Experiment 1., "grammatical" participants). At the conclusion of the task, participants were asked: "What were the grammatical rules or strategies on which you were basing your judgments of grammaticality or classification". No participant was able to accurately identify the rules with any significant success.

\section{Results}

SRT task. The results of one patient with PD were omitted from SRT data analyses because of a technical problem with the microphone and the voicebox, which led to invalid data. Error rates were defined as verbal responses which were incorrect with regard to the position of the stimuli, as well as any omissions. Both groups averaged well below a 5\% error rate across blocks and did not differ significantly in total error rate, $\mathrm{t}(24)=-1.22, \mathrm{p}>.05$. Incorrect responses were not included in the RT analyses. For each set of 10 trials (the sequence pattern in blocks 1 to 4), each participant's median RT of correct responses was computed. Figure 1. shows the mean of those median scores for each block (ten repetitions of 10 trials) for the PD and control groups. All analyses involved a mixed Group x Block ANOVA with Block as a within-group factor. A 2 (Group) x 5 (Block) mixed factor ANOVA showed a significant Group effect, $F(1,24)=6.34, p<.05$, and a significant effect of Block $F(4,96)=11.75, p<.0001$. There was no significant Group x Block interaction, $F(4,96)=0.56, p>.05$.

In order to examine both sequence learning and nonspecific practice effects a 2 (Group) x 4 (Block) ANOVA with repeated measures on the last factor was computed over the first four blocks. This revealed a significant main effect for Block, $\underline{\mathrm{F}}(3,72)=15.89, \underline{\mathrm{p}}<.0001$, and a significant main effect for Group, $\mathrm{F}(1,24)=6.02, \mathrm{p}<.05$. There was no Group x Block interaction, $\underline{\mathrm{F}}(3,72)=0.45, \underline{\mathrm{p}}>.05$.

Decreased RT over the first four blocks can result from both sequence learning and non-specific practice effects. To examine sequence-specific learning, a 2 (Group) by 2 (Block) mixed factor ANOVA was computed for Block 4 and Block 5 . This resulted in main effects for Group, $\mathrm{F}(1,24)=7.72, \mathrm{p}<.05$, and Block, $\underline{F}(1,24)=24.21, \underline{p}<.0001$. There was no Group $\mathrm{x}$ Block interaction, $\underline{\mathrm{F}}(1,24)=0.01, \underline{\mathrm{p}}>.05$. 


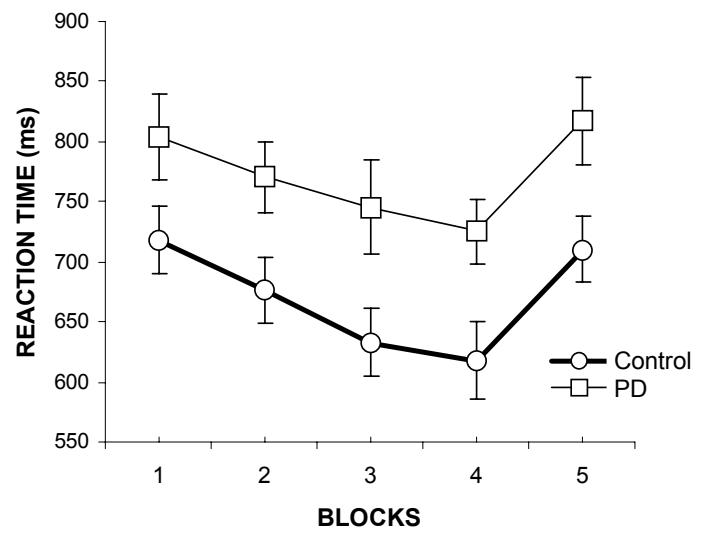

Figure 1. Mean RT across blocks for PD and Control groups.

A preliminary inspection of the effects of disease severity on sequence learning was performed by dividing patients into two groups according to their Hoehn and Yahr scores: Stage 2 $(n=9)$ and Stage 3-4 $(n=3)$. However, the data suggested there was no effect of severity of disease on SRT performance. These results have to be interpreted with caution because of the small number of patients especially in the severe group. A similar pattern of results emerged using both the COWAT and NART as covariates.

Frontal lobe dysfunction has also been associated with impairment in performance on a visuomotor sequence learning task (Beldarrain et al., 1999). However, correlation analysis failed to show a significant association between performance on the COWAT (a test that has been associated with frontal lobe functioning) and sequence specific learning (as measured by the increase in mean reaction time from trial 4 to 5 ) for patients with $\mathrm{PD}, \mathrm{r}=-0.56, \mathrm{p}>.05$. Once again, small numbers preclude any serious conclusions on this matter.

Artificial grammar task. Participants' scores were calculated firstly, by the percentage of grammatical strings classified correctly and the percentage of ungrammatical strings classified correctly, and secondly, by the percentage of grammatical strings classified as grammatical relative to the percentage of grammatical strings classified as ungrammatical.

Average percentage correct for making grammaticality judgments for the patients with PD was 55.9\% (standard error of the mean $(\mathrm{SEM})=2.1 \%$ ), a performance significantly better than chance, $\mathrm{t}(12)=2.85, \mathrm{p}<.01$. Controls obtained $57.9 \%(\mathrm{SEM}=1.9 \%)$ correct for grammaticality judgments, and also performed better than chance, $\underline{\mathrm{t}}(13)=4.32, \underline{\mathrm{p}}<.001$. There was no significant difference in classification performance between the groups, $\underline{\mathrm{t}}(25)=0.75, \underline{\mathrm{p}}>.05$.

Patients with PD classified as grammatical 61.2\% (SEM $=$ $3.4 \%)$ of the grammatical strings and $49.5 \%(\mathrm{SEM}=4.7 \%)$ of the ungrammatical strings. Control participants classified as grammatical $63.4 \%(\mathrm{SEM}=3.9 \%)$ of the grammatical strings and $47.5 \%(\mathrm{SEM}=3.7 \%)$ of the ungrammatical strings. A two-way ANOVA revealed a significant effect of the Grammaticality variable, $\underline{F}(1,25)=25.24, \underline{p}<.0001$, but no significant Group effect, $\underline{\mathrm{F}}(1,25)=0.0003, \underline{\mathrm{p}}>.05$. There was no significant Group $\mathrm{x}$ Grammaticality variable interaction, $\underline{F}(1,25)=0.57, \underline{p}>.05$.

\section{Discussion}

The present study compared the performance of patients with PD with matched controls on two distinct tests of implicit learning, a verbal version of the SRT task and an AG task. Contrary to our first hypothesis the participants with PD demonstrated implicit learning on the SRT task. As predicted, they also showed implicit learning on the AG task. The control group also demonstrated implicit learning on both tasks. These results are further testimony to the robustness of implicit learning in the face of both age (given the mean ages of both groups) and neurological damage. At the same time the failure to observe impaired learning on the SRT task, by the PD participants, is inconsistent with other recent studies (e.g., Jackson et al., 1995; Westwater et al., 1998).

Perhaps the first point to consider is that findings regarding implicit learning and $\mathrm{PD}$ have been quite diverse and sometimes conflicting. For example, findings of deficits in performance of patients with PD on rotor-pursuit tasks (Harrington et al., 1990; Heindel et al., 1989) and mirror reading skill acquisition tasks (Allain et al., 1995; Yamadori et al., 1996) are tempered by findings of preserved learning on both the former (Bondi \& Kasniak, 1991), and the latter (Bondi \& Kasniak, 1991; Harrington et al., 1990). Moreover, attempts to relate findings at a behavioral or cognitive level, with likely neuroanatomical substrates have also produced a complex picture. For example, some authors attribute performance deficits to the disrupted basal ganglia in PD, or argue for a more specific emphasis on brain stem structures of the basal ganglia such as the substantia nigra, or other basal nuclei including the caudate nucleus or the putamen (e.g., Doyon et al., 1997). Others attribute the primary role to impaired neuroanatomical circuitry in PD (e.g., Bondi \& Kaszniak, 1991; Heindel et al., 1989; Taylor, Saint-Cyr, \& Lang, 1986), or more specifically the "complex loop" (e.g., Bondi \& Kaszniak, 1991), whereas some authors have emphasized the importance of disturbed striatofrontal or caudate outflow in PD (e.g., Saint-Cyr, Taylor, \& Lang, 1988). In summary, research on implicit learning in PD has produced conflicting results and also a wide range of possible explanations at the anatomical level.

However, studies employing the SRT task have been generally more consistent. Jackson et al. (1995) reported impairments on a variant of the SRT task in a group of 10 non-demented PD patients compared with healthy controls. Pascual-Leone et al. (1993) reported that patients with PD "achieved procedural knowledge" on the SRT task but at a slower rate than healthy controls. Ferraro et al. (1993) concluded that "there does appear to be some breakdown in implicit learning in non-demented PD individuals...." (p.175). Doyon et al. (1997) observed an impairment late in the sequence acquisition process on a version of the SRT for PD patients with a bilateral striatal-dysfunction. Finally, 
Westwater et al. (1998) using a verbal version of the SRT task found implicit learning was impaired in PD. In summary, the evidence that procedural learning is impaired in PD, at least as measured by the SRT task, is generally more consistent that for other dimensions of implicit learning. In light of the SRT studies reviewed above, it is interesting to speculate as to why the PD patients in the present study demonstrated preserved implicit learning.

One possible explanation for this discrepancy then concerns the verbal version of the SRT task adopted for this experiment. Specifically, the current investigation was structured as to minimize the extent to which deficits displayed by the patient group could be artifacts of bradykinesia, akinesia, and/or motor arrests (symptoms commonly associated with PD), rather than failure to demonstrate implicit learning per se. The present findings suggest that difficulties in executing a motor response may be responsible for the impairment in implicit learning of patients with PD, as gauged by the standard SRT tasks which include an overt motor component in the method of response (e.g., Ferraro et al., 1993, Jackson et al., 1995; Pascal-Leone et al., 1993). However, this line of thought must be viewed with some reservations. Firstly, the SRT task used here replicated that of Westwater et al. (1998) who obtained results which conflict with this study. Secondly, findings of impaired PD patient performance on habit learning tasks that do not include a motor component (Knowlton, Mangels, \& Squire, 1996) strongly suggest that the neostriatum is important not just for motor learning but also for acquiring non-motor dispositions that depend on new associations. Finally, the verbal response retains a motor element in which the deficiency is a salient feature of PD. For instance, bradykinesia has been associated with inappropriate and/or lengthy hesitations and a softening of the voice (becoming less audible), often accompanied by monotonous and hurried speech sounds (Knight, 1992). Therefore, while the exclusion of an overt motor component in the SRT task is useful in light of the motor difficulties experienced by patients with PD, it is by itself unlikely to account for the unexpected preserved learning exhibited by patients with PD in the current investigation.

A second reason that could account for the inconsistent SRT performance of PD patient samples observed in studies involves the possible role played by the frontal lobes in visuomotor sequence learning. Jackson et al. (1995) reported evidence for a procedural learning deficit in PD patients on the SRT task. However, when they compared PD patients who scored poorly on the Wisconsin Card Sorting Test (WCST) (suggesting a degree of frontal lobe dysfunction), with patients who scored normally on this test, the "frontal" group appeared to perform considerably worse than either the "non-frontal" group or the healthy controls. Unfortunately, their small sample size (11 PD patients) precluded a meaningful statistical comparison of these subgroups. Beldarrain et al. (1999) examined SRT learning in 22 (non-PD) patients with unilateral prefrontal lesions and observed that learning was impaired in patients with lesions greater than $2 \mathrm{~cm}$ in diameter. In concluding they argued for the "crucial role of the prefrontal cortex in procedural implicit learning" (p.1859). By contrast, Doyon et al.
(1997), who studied PD patients specifically, concluded that implicit learning depended upon the "integrity of both the striatum and the cerebellum, but not of the frontal lobes" (p.219). In the present study, pairwise correlations between COWAT performance and sequence specific learning on the SRT failed to reach significance supporting Doyon and colleagues' findings. However, these results must be interpreted with caution given that numbers were small and the COWAT is a measure of verbal fluency and not frontal lobe integrity per se. Future research would be advised to adopt more precise measures of frontal lobe functioning such as the WCST. In summary, there is some evidence, although far from unequivocal, that the intact functioning of the prefrontal cortex may be important for procedural learning. If this can be substantiated, then it has obvious relevance for clarifying the performance of PD patients on the SRT task, given that "frontal dysfunction" is such a common symptom of PD (Taylor et al., 1986).

A third possible explanation for the inconsistent findings in this area concerns the stage of the disease. Presumably, if the implicit learning deficit is related to damage to the basal ganglia, then this will become increasingly obvious as the disease advances. In support of this Doyon et al. (1997) found that "only PD patients in more advanced stages of the disease showed an impairment in acquiring the repeating sequence" (p.235). Similarly, on the rotary pursuit task, also an example of implicit motor skill learning, Harrington et al. (1990) reported that procedural learning was impaired but only in patients with more advanced symptoms of PD. Interestingly, in the present study, a preliminary analysis of severity did not show any effect, although as 9 of the 12 patients were in Stage 2 on the Hoehn and Yahr scale, this is perhaps not surprising. Another, preferably continuous, measure of motor function or severity that allowed for a more even distribution of the subjects into two groups would have perhaps been more useful given the small number of patients.

Finally, it is important to note that implicit learning on the AG task was also preserved among the PD patients. Overall, both groups classified strings according to their grammatical status at a level above chance, demonstrating learning for the AG system, learning that could not be consciously articulated by participants in either group. These findings are consistent with those of both Reber and Squire (1999) and Thierry et al. (1998) who also observed preserved AG learning in patients with PD and is in accord with current notions that such learning of perceptual knowledge is more cortically mediated and less reliant upon subcortical structures (Reber \& Squire, 1999). Though we have devoted most of the discussion to considering explanations for the unpredicted results on the SRT task, the results on the AG task are also important, as this is only the third published study to date reporting preserved implicit learning on this task in PD patients. As such it adds to the growing body of evidence for the robustness of this dimension of implicit learning even in the face of neurological illness. 


\section{References}

Allain, H., Lieury, A., Quemener, V., Thomas, V., Reymann, J., \& Gandon, J. (1995). Procedural memory and Parkinson's disease. Dementia, 6, 174-178.

Beldarrain, M. G., Grafman, J., Pascual-Leone, A., \& Garcia-Monco, J. C. (1999). Procedural learning is impaired in patients with prefrontal lesions. Neurology, 52, 1853-1860.

Benton, A. L., \& Hamsher, K. (1976). Multilingual aphasia examination. Iowa City: University of Iowa.

Bondi, M. W., \& Kasniak, A. W. (1991). Implicit and explicit memory in Alzheimer's disease and Parkinson's disease. Journal of Clinical and Experimental Neuropsychology, 13, 339-358.

Dienes, Z., Broadbent, D., \& Berry, D. (1991). Implicit and explicit knowledge bases in AG learning. Journal of Experimental Psychology: Learning, Memory, and Cognition, 17, 875-887.

Doyon, J., Gaudreau, D., Laforce, R., Castonguay, M., Bedard, P. J., Bedard, F., \& Bouchard, J-P. (1997). Role of the striatum, cerebellum, and frontal lobes in the learning of a visuomotor sequence. Brain and Cognition, $34,218-245$

Ferraro, F. R., Balota, D. A., \& Connor, L. T. (1993). Implicit memory and the formation of new associations in nondemented Parkinson's disease individuals and individuals with senile dementia of the Alzheimer's type: A serial reaction time investigation. Brain and Cognition, 21, 163-180.

Folstein, M. F., Folstein, S. E., \& McHugh, P. R. (1975). "Mini-Mental State": A practical method for grading the cognitive state of patients for the clinician. Journal of Psychiatric Research, 12, 189-198.

Harrington, D. L., Haaland, K. Y., Yeo, R. A., \& Marder, E. (1990). Procedural memory in Parkinson's disease: Impaired motor but not visuo-perceptual learning. Journal of Clinical and Experimental Neuropsychology, 12, 323-339.

Heindel, W. C., Salmon, D., Shults, C., Walicke, P. A., \& Butters, N. (1989). Neuropsychological evidence for multiple implicit memory systems: A comparison of Alzheimer's, Huntington's and Parkinson's disease patients. Journal of Neuroscience, 9, 582-587.

Hoehn, M. M., \& Yahr, M. D. (1967). Parkinsonism: Onset, progression, and mortality. Neurology, 17, 427442.

Howard, D. V., \& Howard, J. H., Jr. (1989). Age differences in learning serial patterns: Direct versus indirect measures. Psychology and Aging, 4, 357-364.

Jackson, G. M., Jackson, S. R., Harrison, J., Henderson, L., \& Kennard, C. (1995). Serial reaction time learning and Parkinson's disease: Evidence for a procedural learning deficit. Neuropsychologia, 33, 577-593.

Knight, R. G. (1992). The neuropsychology of degenerative disorders. Hillsdale, NJ: Lawrence Erlbaum.

Knopman, D., \& Nissen, M. J. (1987). Implicit learning in patients with probable Alzheimer's disease. Neurology, 37, 784-788.
Knowlton, B. J., Mangels J. A., \& Squire, L. R. (1996). A neostriatal habit learning system in humans. Science, 273, 1399-1402.

McDowall, J. \& Martin, S. (1996). Implicit learning in closed-head-injured subjects: Evidence from an event sequence learning task. New Zealand Journal of Psychology, 25, 2-6.

Nelson, H. E., \& Willeson, J. R. (1991). National Adult Reading Test (2nd ed.). Windsor, UK: NFER-Nelson.

Nissen, M. J., \& Bullemer, P. (1987). Attentional requirements of learning: Evidence from performance measures. Cognitive Psychology, 19, 1-32.

Pascual-Leone, A., Grafman, J., Clark, K., Stewart, M., Massaquoi, S., Lou, J., \& Hallett, M. (1993). Procedural learning in Parkinson's disease and cerebellar degeneration. Annals of Neurology, 34, 594-602.

Reber, A. S. (1989). Implicit learning and tacit knowledge. Journal of Experimental Psychology: General, 118, 219235.

Reber, P. F., \& Squire, L. R. (1999). Intact learning of AGs and intact category learning by patients with Parkinson's disease. Behavioral Neuroscience, 113, 235-242.

Saint-Cyr, J. A., Taylor, A. E., \& Lang, A. E. (1988). Procedural learning and neostriatal dysfunction in man. Brain, 111, 941-959.

Seger, C. A. (1998). Multiple forms of implicit learning. In M. A. Stadler, and P. A. Frensch (Eds.), Handbook of implicit learning. London: Sage.

Squire, L. R. (1994). Declarative and nondeclarative memory: Multiple brain systems supporting learning and memory. In D. L. Schacter, and E. Tulving (Eds.), Memory systems. Cambridge, Mass.: MIT Press.

Squire, L. R., \& Zola, S. M. (1996). Structure and function of declarative and nondeclarative memory systems. Proceedings of the National Academy of Sciences, 93, 13515-13522.

Taylor, A. E., Saint-Cyr, J. A., \& Lang, A. E. (1986). Frontal lobe dysfunction in Parkinson's disease. Brain, 109, 845-883.

Thierry, M., Peigneux, P., \& Van der Linen, M. (1998). Preserved AG learning in Parkinson's disease. Brain and Cognition, 37, 109-112.

Westwater, H., McDowall, J., Siegert, R., Mossman, S., \& Abernethy, D. (1998). Implicit learning in Parkinson's disease: Evidence from a verbal version of the serial reaction time task. Journal of Clinical and Experimental Neuropsychology, 20, 413-418.

Yamadori, A., Yoshida, T., Mori, E., \& Yamashita, H. (1996). Neurological basis of skill learning. Cognitive Brain Research, 5, 49-54. 